\title{
La cultura histórica humanista y renovadora en "La edad de oro" de José Martí
}

\author{
"The humanist and renovating historical culture in \\ "The age of gold" of José Martí
}

\section{Resumen}

El trabajo que se presenta, forma parte de los resultados de la actividad investigativa que se desarrolla en el proyecto científico Educar con José Martí en la escuela cubana, de la Universidad de Matanzas. La Edad de Oro (1959), reconocida en el siglo XXI como un texto científico de naturaleza interactiva y participativa, por la profundidad de su contenido, el humanismo de su prosa y la estimulación hacia el seguimiento en el tiempo de lo que en ella se aborda, constituye una obra de necesaria consulta en las diferentes etapas de la vida. Se fundamenta la concepción de la cultura histórica humanista y renovadora en La Edad de Oro de José Martí, como referente metodológico de la preparación de los docentes de la enseñanza general.

Palabras clave: José Martí, La Edad de Oro, cultura histórica.

\begin{abstract}
The work that is presented is part of the results of the investigative activity that is developed in the scientific project to Educate with José Martí in the Cuban school, of the University of Matanzas. The Age of Gold (1959), recognized in the XXI century as a scientific text of interactive nature and participativa, for the depth of its content, the humanism of its prose and the stimulation toward the pursuit in the time of what is approached in her, constitutes a text of necessary consultation in the different stages of the life. The conception of the humanist and renovating historical culture is based in The Age of Gold of José Martí like relating methodological of the preparation of the educational ones of the general teaching.
\end{abstract}

Keywords: José Martí, The Age of Gold, historical culture.

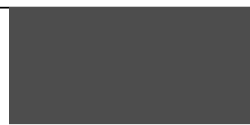

\section{Correspondencia de autor:}

yuseli.pestana@umcc.cu

Recibido:

$17 /$ Marzo/2019

Aceptado:

4/Mayo/2019

Publicado:

Cómo citar este artículo:

Pestana Llerena, Y. (2019). La cultura histórica humanista y renovadora en "La edad de oro" de José Martí. Revista Amauta, 17(34), pp.9-24. Dol: http://dx.doi.org/10.15648/am.34.2019.2 


\section{Introducción}

La dirección orientadora que representa el estudio del legado de la obra de José Martí, para la educación de las presentes y futuras generaciones de cubanos, permite encontrar el equilibrio entre la espiritualidad y la conciencia que emanan de su pensamiento y acción y la objetividad en el análisis de hechos, procesos, fenómenos y personalidades de la historia universal y de Cuba. De ahí la necesidad de comprender el ámbito martiano, fuentes para su estudio y resortes estimuladores de ciencia y conciencia en el desarrollo de su fecunda vida y obra. Para una mejor comprensión de lo expresado, resulta útil el conocimiento de la misma desde una visión sincrónica (Pestana, 2012).

Hombre de naturaleza contestataria ante la servidumbre social del siglo XIX, la esclavitud y el despojo humano, de pensamiento agudo y proyectivo, crítica consciente, inteligencia notable y elegancia en el trato, "atrajo a su derredor a las almas jóvenes y levantadas, a escritores y poetas, a tribunos y filósofos, a cuanto daban, lozano y vivo, como flor de regeneración y fruto venidero de grandeza, aquellas tierras" (Bolet, 1971, p.19). Tales evocaciones advierten el carácter progresista de su pensamiento entorno a la política, la educación y la cultura de la segunda mitad del siglo XIX y lo ubican en la esencia de la nacionalidad y nación cubanas.

La Edad de Oro (1959), una de sus obras cumbres, reconocida en el siglo XXI como un texto científico de naturaleza interactiva y participativa, por la profundidad de su contenido, el humanismo de su prosa y la estimulación hacia el seguimiento en el tiempo de lo que en ella se aborda, constituye un texto de necesaria consulta en las diferentes etapas de la vida.

Con un sentido renovador y estimulador de nuevos enfoques para su estudio, aprendizajes y de un potencial educativo en correspondencia con la cultura y la efectividad en los desempeños formativos de los educadores cubanos, trasciende al siglo XXI en medio de nuevas complejidades para la sociedad, la cultura, la ciencia y la educación. De ahí que los educadores cubanos del, siglo XXI mantengan vivo el patrimonio inmaterial esencial que La Edad de Oro representa: símbolo de conciencia nacional y motivación para la formación en el respeto hacia la condición humana, principalmente desde la escuela como agencia socializadora de la educación. Constituye el 
objetivo del trabajo: fundamentar la concepción de la cultura histórica humanista y renovadora en La Edad de Oro de José Martí como referente metodológico de la preparación de los docentes de la enseñanza general.

\section{Desarrollo}

La condición educable del ser humano, presupone el desarrollo de múltiples y variados procesos formativos a lo largo de toda la vida, que responden a objetivos y fines en correspondencia con la cultura de su tiempo o particularmente a la influencia derivada del paso por cada uno de los niveles educativos. En el siglo $\mathrm{XXI}$, al cierre de la segunda década, la educación cubana demanda el desarrollo de proyectos de diversa naturaleza: educativos, de investigación, socioculturales y comunitarios, que enriquezcan los modos de hacer, sentir y transformar el empleo de la obra martiana como asidero indispensable de la educación y por tanto, fuente de inspiración y transformación.

Considerada "Única en el género de literatura infantil fue sin duda la revista La Edad de Oro, editada por A[aron] Dacosta Gómez, el generoso amigo de Marti" (J.M.O.C., T-27, 1975, p.157). Los cuatro números publicados aparecieron en julio, agosto, septiembre y octubre de 1989, en Nueva York y fueron redactados por José Martí.

La revista contribuyó al conocimiento de la historia, la ciencia y la sociedad con un lenguaje lleno de "ternura, y amor para los niños" (J.M.O.C., T-27, 1975, p.157). La filosofía de la educación, la concepción de la historia, el potencial educativo que posee, así como la cultura histórica que se identifican e irradian sus páginas, permiten considerar a La Edad de Oro como una obra con plena vigencia en el siglo $X X I$, por lo que ha sido fuente de inspiración para la escritura de nuevos textos como Pedagogía de la ternura (2002) de Lidia Turner Martí y Balbina Pita Céspedes.

Al decir de Justo Chávez, quien prologara el referido texto:

La Pedagogía de la ternura se basa en los postulados educativos martianos.

Lidia y Balbina han penetrado a fondo en el pensamiento del Apóstol y han extraído bien la savia nutricia de su teoría educativa, la han 
hilvanado con hilo y aguja mágicos y han logrado el milagro de una alternativa pedagógica diferente. (Chávez, 2002, s/p)

Entre los textos de mayor comprensión de aspectos sobre la historia, la política, la literatura y la ciencia de La Edad de Oro se encuentran: "Tres Héroes", donde se presentan semblanzas de la vida de Bolívar, San Martín e Hidalgo; "La llíada de Homero"; "La historia del hombre contada por sus casas"; "Las ruinas indias"; "Músicos, poetas y pintores"; "La Exposición de Paris" y "El Padre Las Casas". Y de muy honda ternura y generoso sentido humano son: su cuento "La Muñeca Negra", sus famosos versos "Los zapaticos de rosa" y La Edad de Oro.

Con la motivación de dotar a docentes y directivos de la educación general de un texto en el cual se precisen los elementos para el desarrollo de una cultura histórica humanista y renovadora en los educandos desde el estudio de La Edad de Oro de José Martí, es necesario tener en cuenta los componentes que la estructuran. En la Figura 1 se presentan a continuación:

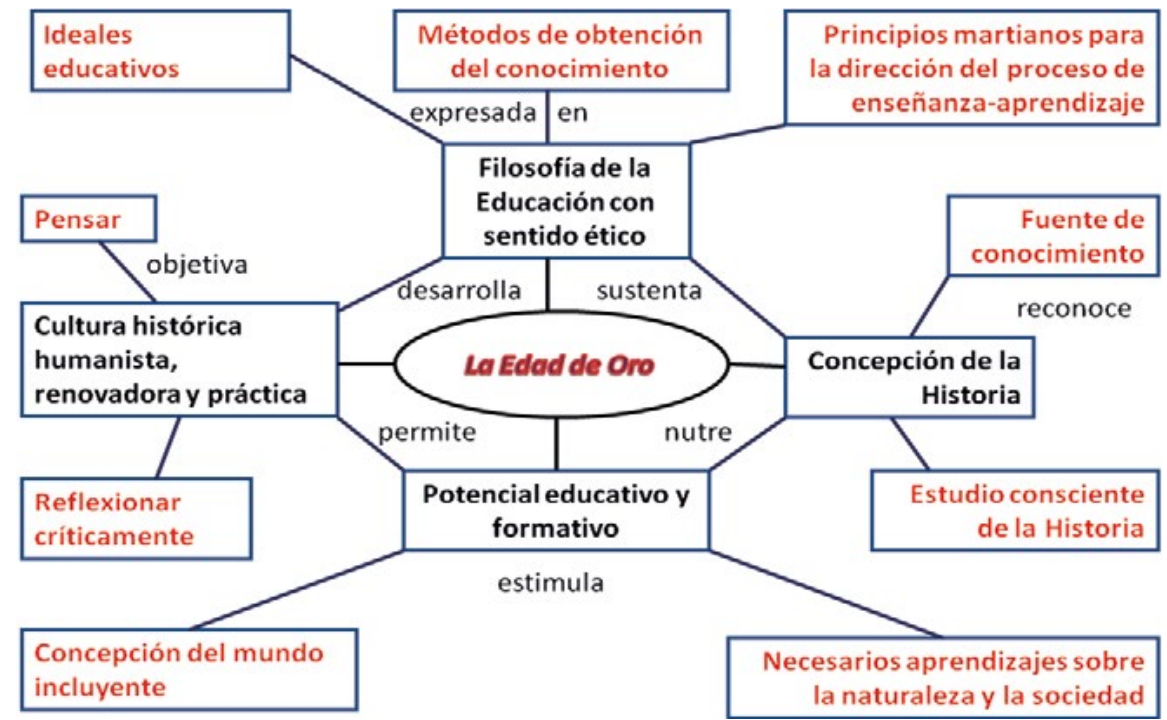

Figura 1. Componentes para el desarrollo de una cultura histórica humanista y renovadora desde el estudio de La Edad de Oro de José Martí

Fuente: Tomado de Sabariego (2002, p.96) 
El estudio del legado de José Martí al conocimiento de la historia como pauta para el desarrollo de una cultura histórica, implica reconocer en su base la existencia de una filosofía de la educación humanista con un profundo sentido ético. Identificada con la educación del ser humano para la vida y con la utilidad de la virtud. Es la filosofía de la educación humanista martiana el soporte ético de referencia de una actitud hacia la educación continua y ascendente del ser humano que no culmina sino con la muerte.

Ante una evocación de tal magnitud, aparecida en su teoría educativa cuales "..."críticas", "elogios", "consideraciones ocasionales", "consejos sembrados", "aseveraciones absolutas" que en forma de aforismos revelan las esencias de su pensamiento filosófico educativo" (Martínez, 2003, p.19), se pueden determinar los resortes ético-humanistas en su filosofía de la educación, entre ellos: la unidad entre lo cognitivo y lo afectivo, la necesidad de educar la inteligencia a partir de la ciencia como algo natural, la defensa de la identidad latinoamericana y la vinculación del estudio con el trabajo.

Como parte de su concepción de la educación (Escribano, 2006), expresión de su pensamiento educativo auténtico y renovador, para la segunda mitad del siglo XIX, pueden identificarse algunas generalizaciones que, a la luz del desarrollo de la didáctica del siglo XXI, representan o advierten principios de la enseñanza. De igual forma, sus métodos e ideales educativos, ocupan no pocas reflexiones y valoraciones, ambos han servido de pautas para la enseñanza de materias como la Historia y la Literatura, aunque las ciencias también se nutren del legado martiano. Desde la perspectiva del Apóstol "Nada sugiere tanta y tan hermosa Literatura como un párrafo de ciencia" (J.M. 0.C., T-22, 1975, p.141).

En Cuba, por solo citar un ejemplo de principio de enseñanza basados en la obra de José Martí, existen como antecedentes los trabajos del profesor Horacio Díaz Pendás, quien determinó según su criterio y experiencia, Cinco pautas martianas para el profesor de Historia, las cuales son: Adecuar la enseñanza a las necesidades y particularidades del alumno, Lograr la vitalidad de la palabra oral en su oficio de comunicarse con animación y entusiasmo, Convertir el diálogo en un método imprescindible en el camino del convencimiento, Indagar constantemente en la labor 
del alumno, y El "enseñar como sin querer" (Díaz, 1999, pp.161-175). Se trata, dicho con la inspiración del magisterio martiano, de

enseñar a razonar, para propiciar la labor colectiva, para andar juntos educador y educandos el camino del conocimiento y del enriquecimiento humano, no podría hacerse de espaldas a una realidad y a su enjuiciamiento crítico, por muy "apolítico" que quisiera ser un pedagogo. (Díaz, 2006, p.44)

Es por ello que concebir la dirección del proceso de enseñanza-aprendizaje que favorezca el desarrollo y comprensión de la naturaleza ética martiana, es una necesidad y motivo de inspiración de las presentes y futuras generaciones de cubanos. Ante esta demanda social, en condiciones de construcción e institucionalización de una sociedad cada vez más justa e incluyente para todos los cubanos, se socializa un sistema de principios martianos para la dirección del proceso de enseñanza-aprendizaje que a su vez, revelan una concepción de la historia, del mundo, de la sociedad y del ser humano.

Con ellos se intenta ofrecer un punto de partida para el empleo de La Edad de Oro como fuente de conocimiento y estudio de la historia. Para lograr una correcta aplicación de estos principios, se debe trabajar de forma articulada con la familia y la comunidad como agencias socializadoras de la educación, bajo la orientación de la escuela y los agentes socializadores especializados. Es por ello que aprender de José Martí y con él, no tiene espacio restringido.

Para el hombre de La Edad de Oro, fue evidente la necesidad de formar un vínculo de la teoría con la práctica' y que la escuela debía desarrollar un hombre vivo e independiente. En este aspecto, no solo determina, se apropia y pone en práctica las claves, constantes o postulados heredados de ilustres maestros como Félix Varela y José de la Luz y Caballero, sino que va más allá: Ios enriquece y hace de ellos el reto de su tiempo. Este enriquecimiento lo realizó desde la cultura y para la cultura.

En uno de sus artículos en La Edad de Oro, escribió:

En el mismo hombre suelen ir unidos un corazón pequeño y un talento grande. Pero todo hombre tiene el deber de cultivar su inteligencia, por

1 Los subrayados expresan el criterio de los autores del trabajo. 
respeto a sí propio y al mundo. Lo general es que el hombre no logre en la vida un bienestar permanente sino después de muchos años de espera con paciencia y de ser bueno, sin cansarse nunca. El ser bueno da gusto, y lo hace a uno fuerte y feliz. (J.M. 0.C., T-18, 1975, pp.390-91)

El conocimiento era para él mucho más que el simple acceso a la cultura, era la base más importante para la formación de valores, sentimientos, convicciones y virtudes de su personalidad, con un fuerte contenido ético. En su obra, lo cognoscitivo marcha orgánicamente vinculado a lo axiológico. En este sentido expresó: "Las cualidades morales suben de precio cuando están realzadas por cualidades inteligentes", y más adelante apuntó:

El pueblo más feliz es el que tenga mejor educados a sus hijos en la instrucción del pensamiento, y en la dirección de los sentimientos. (...) Un pueblo virtuoso vivirá más feliz y más rico que otro lleno de vicios y se defenderá mejor de todo ataque. (J.M. 0.C., T-19, 1975, p.375)

Conocer la Historia de los pueblos americanos, sus costumbres, tradiciones, motivos de luchas, es una manera de identificarse con las posibles soluciones a sus problemas contemporáneos y trabajar por ellas.

En la intención de promover la colaboración, la fraternidad y la igualdad, José Martí concibió las relaciones, en el contexto de la clase, como un intercambio, no solo entre el profesor y los educandos, sino también entre estos últimos. La forma de impartir cada materia, el respeto, la igualdad que preside cada encuentro, revela el grado de compromiso y de hermandad para dar a conocer cada idea.

Un ejemplo de su labor en este sentido, está contenido en su descripción del trabajo conjunto de profesores y alumnos en La Liga:

La Liga es escuela de letras necesarias (...) Uno enseña aritmética viva, y descompone los números para que se les vean los goznes, que es mejor modo que el de meras reglas. Otro, con la mano que estuvo en la gran gloria, guía al hombre hecho que viene a pedir letra. Otro, en conversación ambulante, y manteniendo lo uno con lo demás, trata de los primeros conocimientos, y pica al principiante la curiosidad mayor. Otro se sienta a la mesa de preguntas, llena de escritos sin firma, y va 
hablando sobre cada cual de ellos, responde al tema, nota los méritos del escritor, endereza las faltas, predica la sinceridad de la forma, que enaltece el carácter tanto como lo vicia, sin sentir, la forma insincera. Otro es gramático de obras, que pone y descompone ante los ojos el artificio del lenguaje, de modo que como quiera que caiga la frase quede en pie, y a las palabras les busca la historia y el parentesco, que es la escuela para quien anhela pensar bien. Detrás del maestro, abierta a todos, está la librería, en sus estantes de color de luz. (J.M.O.C., 1975, $T-5$, p.254)

A partir de esta descripción, se revela una esencia: el empleo de métodos participativos que garanticen el desarrollo de razonamientos individuales y grupales. Esta dinámica de trabajo favorece el cumplimiento de un rol efectivo en el contexto grupal, estimula la participación y a su vez, desarrolla la capacidad de escuchar las diferentes formas de pensar y los argumentos que las sostienen. Además, estimula la participación, pues cada individuo tiene la oportunidad de aprender y aportar desde la potencialidad que lo identifica.

Para Cintio Vitier, martiano de prosa y espíritu, "La pedagogía martiana está en Martí. Él es el que, página por página, nos enseña a enseñarlo. En La Liga practicó el método conversacional, que elogió en Sócrates y en Bronson Alcott, que aconsejó a los "maestros ambulantes". La Edad de Oro, ¿qué es sino escritura de la oralidad, una conversación que no termina?" (Vitier, 2000, p.33). De esta forma, son los principios martianos enunciados generales, puntos de partida que se articulan y convergen en una concepción del mundo incluyente y de constantes posibilidades y aperturas para nuevos y cada vez más necesarios aprendizajes sobre la naturaleza y la sociedad.

Los principios martianos para la dirección del aprendizaje, constituyen el reflejo de una filosofía de la educación que subyace en la magnitud de su legado y que se revelan en La Edad de Oro, específicamente en la educabilidad del ser humano y en la utilidad de la virtud; en la intención de "decirles a los niños lo que deben saber para ser de veras hombres. Todo lo que quieran saber (...) y de modo que lo entiendan bien..." (J.M.O.C., T-18, 1975, p.301); en la capacidad de reconocer cuando "un niño puede ser hermoso aunque sea feo; un niño bueno, inteligente y aseado es siempre 
hermoso" (J.M.O.C., T-18, 1975, p.301) y en la formación de "hombres que digan lo que piensan y lo digan bien: hombres elocuentes y sinceros" (J.M.0.C., T-18, 1975, p.303).

Estos criterios esbozan nociones antrológicas, epistemológicas, metodológicas, axiológicas y teleológicas, por lo que la filosofía de la educación martiana expresada en La Edad de Oro, sustenta el desarrollo de una concepción de la historia en la que esta es fuente de conocimiento y por tanto demanda un estudio consciente.

La Historia, en esencia es la ciencia que estudia el papel de las masas y las personalidades en el devenir social, el desarrollo de hechos, procesos y fenómenos concatenados y condicionados por relaciones causales, temporales y espaciales. Este aprendizaje aunque signado por la fuerza transformadora de los planes y programas de estudio, puede llegarse a él desde el interés por el origen de las cosas, descubrir las relaciones, nexos inherentes a procesos que tienen lugar en la naturaleza, la sociedad y en el propio pensamiento del ser humano.

Desde la pedagogía martiana, La Edad de Oro contribuye al conocimiento de la historia y se publica

para que los niños americanos sepan cómo se vivía antes, y se vive hoy, en América, y en las demás tierras; y cómo se hacen tantas cosas de cristal y de hierro, y las máquinas de vapor, y los puentes colgantes, y la luz eléctrica; para que cuando el niño vea una piedra de color sepa por qué tiene colores la piedra, y qué quiere decir cada color; para que el niño conozca los libros famosos donde se cuentan batallas y las religiones de los pueblos antiguos. (J.M.0.C., T-18, 1975, p.301)

Aunque José Martíno elaboró conscientemente una concepción sistémica y orgánica sobre la historia, en la amplitud de su obra aparecen esencias que revelan la misma. Los siguientes trabajos constituyen solo una muestra de ello: A La Colonia Española (J.M.O.C., T-1, 1975); "Placeres y problemas de septiembre" (J.M.O.C., T-10, 1975); "El General Grant" (J.M.O.C., T-13, 1975); "Italia" (J.M.O.C., T-14, 1975); "España" (J.M.O.C., T-14, 1975); Libros nuevos (J.M.O.C., T-15, 1975); Cuadernos de Apuntes (J.M.O.C., T-21, 1975); Fragmentos (J.M.O.C., T-22, 1975) y Serie de artículos para "La América" (J.M.O.C., T-23, 1975).

En la concepción de la historia esbozada por José Martí en La Edad de Oro, sobresalen las siguientes esencias: el estudio del pasado como cultura de la humanidad en las diferentes regiones del mundo, símbolos de idiosincrasia y diversidad; el estudio de 
los hechos, procesos, fenómenos y adelantos científicos que rodean al ser humano como cultura de su tiempo, y el placer de conocer para emprender y transformar.

En resumen, la concepción martiana de la historia nutre el potencial educativo y formativo cimentado en La Edad de Oro. En el Cuadro No.1 que aparece a continuación, se presenta un sistema de preguntas y respuestas que ilustran cómo pudiera abordarse un diálogo entre el texto de José Martí y los niños y cómo el propio Apóstol con profunda sensibilidad y sencillez, llegó a idealizar un posible encuentro con un niño de "Nuestra América".

Cuadro 1. Preguntas y respuestas que estimulan el acercamiento afectivo a José Martí. (J.M.O.C., T-18, 1975, pp.301-303)

\begin{tabular}{|c|c|}
\hline Preguntas & Respuestas \\
\hline ¿Con qué fin se publica La Edad de Oro? & "Para conversar una vez al mes" (p.301) \\
\hline ¿Cómo nos reconoceremos? & "como buenos amigos" (p.301) \\
\hline ¿Con quiénes conversaremos? & "con los caballeros de mañana, y con las madres de mañana" (p.301) \\
\hline ¿De qué conversaremos? & $\begin{array}{l}\text { De "todo lo que han hecho los hombres hasta ahora" (p.301); } \\
\text { "cuentos de risa y novelas de niños, para cuando hayan } \\
\text { estudiado mucho, y quieran descansar" (p.302) }\end{array}$ \\
\hline ¿Qué queremos? & "Oue nos quieran" (p.302) \\
\hline ¿Cómo queremos que nos vean? & "Como cosa de su corazón" (p.302) \\
\hline ¿Cómo nos comunicaremos? & $\begin{array}{c}\text { "escríbanos como si nos hubiera conocido siempre, que } \\
\text { nosotros le contestaremos" (p.302) }\end{array}$ \\
\hline ¿Y si no sabemos escribir bien todavía? & $\begin{array}{l}\text { "No importa que la carta venga con faltas de ortografía. Lo } \\
\text { que importa es que el niño quiera saber" (p.302) }\end{array}$ \\
\hline ¿Y si la carta está bien escrita? & $\begin{array}{l}\text { "La publicaremos en nuestro correo con la firma al pie, } \\
\text { para que se sepa que es niño y que vale" (p.303) }\end{array}$ \\
\hline ¿Cómo puedo escribir de algo sobre mi edad? & "para escribir bien de una cosa hay que saber de ella mucho"(p.303) \\
\hline ¿Qué deben saber las niñas y los niños? & $\begin{array}{l}\text { "Las niñas deben saber lo mismo que los niños, para poder } \\
\text { hablar con ellos como amigos cuando vayan creciendo" (p.303) }\end{array}$ \\
\hline ¿Qué hay de particular en las niñas? & $\begin{array}{l}\text { "en las almas de las niñas sucede algo parecido a lo que } \\
\text { ven los colibríes cuando andan curioseando por entre las } \\
\text { flores. Les diremos cosas así, como para que las leyesen } \\
\text { los colibríes, si supiesen leer." (p.303) }\end{array}$ \\
\hline $\begin{array}{c}\text { Después de leer La Edad de Oro, ¿qué } \\
\text { queremos? }\end{array}$ & "que los niños sean felices" (p.303) \\
\hline $\begin{array}{l}\text { ¿Y"si alguna vez nos encuentra un niño } \\
\text { de América por el mundo"? }\end{array}$ & $\begin{array}{l}\text { Que "nos apriete mucho la mano, como a un amigo viejo, } \\
\text { y diga donde todo el mundo lo oiga: "iEse hombre de La } \\
\text { Edad De Oro fue mi amigo!"'”(p.303) }\end{array}$ \\
\hline
\end{tabular}


A la luz del siglo XXI, es posible reconocer el potencial educativo y formativo implícito en la obra martiana, aspecto dinamizador del desarrollo de una concepción del mundo incluyente, que condiciona la búsqueda de necesarios aprendizajes sobre la naturaleza y la sociedad. Se trata de que todos participen porque "Para los niños es este periódico, y para las niñas, por supuesto. Sin las niñas no se puede vivir, como no puede vivir la tierra sin luz", y "todo lo que quieran saber les vamos a decir" (J.M.0.C., T-18, 1975, p.301). Estas son claves martianas, con un didactismo tal, que los niños pueden comprender con facilidad lo que se quiere decir, cómo se dice y para qué se dice.

Desde una percepción adulta, se puede plantear que en La Edad de Oro hay Filosofía, Historia, Cultura, Ciencia, Literatura y Política en el entramado del conocimiento, hay una forma de decir que enamora y compromete porque hay una ternura en la pedagogía que revela. Además, provoca un impacto en el lector que deviene en una relación fantasía-realidad que cada individuo la objetiva en correspondencia con su cultura, con la manera de concebir las relaciones sociales, la ciencia y el ser humano.

El potencial educativo y formativo que encierra La Edad de Oro, permite desde el conocimiento de la historia, el desarrollo de una cultura histórica (Pestana, Fajardo, Menéndez, E. \& Diago, 2015), humanista y renovadora en los educandos. Ello implica necesariamente reconocer un sistema de relaciones, entre: el significado del desarrollo de la cultura histórica; cómo se concibe la cultura histórica en el contexto universitario y qué lugar ocupa en el desarrollo de la cultura general integral y la determinación de la obra martiana como un medio para el desarrollo de la cultura histórica, entre los aspectos esenciales.

El término cultura histórica en esencia, advierte la integración de valores, formación de juicios, criterios y valoraciones en los individuos, al tomar como base el conocimiento histórico y la problematización que ello implica.

Es La Edad de Oro, un ejemplo de educación de la cultura histórica en las nuevas generaciones de cubanos. La nueva cualidad favorece, desde el encuentro con el pasado, el conocimiento de todo lo que ha realizado el ser humano en determinadas condiciones geográficas, históricas, sociales y económicas, en las que se ha desarrollado y que han condicionado el surgimiento y desarrollo de ideas políticas, filosóficas, éticas, estéticas e ideológicas. Así se forja el respeto por la historia y 
las tradiciones de otros pueblos del mundo, para encontrar relaciones, conexiones entre el mundo, el continente y los individuos.

En el trabajo "Tres Héroes", se evidencia una profunda reflexión sobre el rol de los seres humanos en la historia: "Esos son héroes; los que pelean para hacer a los pueblos libres, o los que padecen en pobreza y desgracia por defender una gran verdad. Los que pelean por la ambición, por hacer esclavos a otros pueblos, por tener más mando, por quitarle a otro pueblo sus tierras, no son héroes, sino criminales" (J.M.O.C., T-18, 1975, p.308). Este fragmento, además, muestra el respeto a la dignidad humana, el valor del sacrificio a toda prueba, la diferencia entre las acciones que construyen y la ambición, el verdadero sentido de la vida. Su vida fue un ejemplo en este sentido.

Aseveraciones similares, con la intencionalidad de provocar una mayor riqueza de pensamiento, pueden desarrollar los educandos debido a la capacidad de pensar y reflexionar críticamente que el desarrollo de la cultura histórica condiciona, estimula y permite objetivar.

La cultura histórica contribuye, en gran medida, a la formación integral de los educandos, pues refuerza el patriotismo y el antimperialismo. En esa dirección, la contextualización de los procesos de la historia nacional y universal resulta fundamental, como base de una cultura histórica general, que se integra a la importancia que reviste el conocimiento de la obra de José Martí dentro de la contribución al conocimiento de la historia. La cultura histórica, que se objetiva a partir de la lectura de los textos de La Edad de Oro en una vuelta al punto de partida, permite el desarrollo de una filosofía de la educación desde la ética martiana.

Sin embargo, faltaría en el análisis el sentido (humanista), alcance (renovador) y expresión (práctica) que hacen de la cultura histórica el asidero para el desarrollo de una filosofía de la educación que estimula el conocimiento y la formación integral del ser humano desde la ternura, la Ciencia, la Historia, el Arte y la Literatura.

El sentido humanista en la presentación del contenido de los trabajo de La Edad de Oro, tiene como antecedentes el estudio de "los puntos determinantes de la historia" (J.M.O.C., T-21, 1975, p.76), aquellos hechos que marcan giros en la historia de los pueblos como las guerras de conquista; "la común contemplación de las obras 
hermosas" (J.M.0.C., T-14, 1975, p.392), la necesidad de la hermandad, solidaridad, deseos y necesidad de unirse por todo aquello que los proteja, cultive y acreciente su condición humana; la intención de que "se salven todos"(J.M.O.C., T-8, 1975, p.185), entendido como el ponerse en lugar del otro y comprender su sufrimiento. Además, en etapa posterior a la redacción de la revista, resalta el convencimiento del Apóstol acerca de "las leyes de la humanidad". En este aspecto, mucho pudiera comentarse, pero las claves martianas hablan por sí solas "La cobardía y la indiferencia no pueden ser nunca las leyes de la humanidad" (J.M.O.C., T-18, 1975, p.337).

El alcance renovador en la comprensión de los hechos y procesos históricos en La Edad de Oro, tiene como premisa el reconocimiento del rol de la ciencia en la sociedad y su carácter creador. Sin embargo, alerta sobre el peligro que representan las guerras que acaban con todo y la responsabilidad del ser humano como decisor de los procesos de aceleración y desaceleración social y político. La siguiente idea así lo confirma: "Palabras sobre ciencia borran la impresión desagradable que produce emplear la inteligencia creadora en ideas sobre destrucción. Imitarán a Bárcena muchos mexicanos; la patria estaría más orgullosa con los hijos que la honran que no con los que la ensangrientan" (J.M.O.C., T-6, 1975, p.257).

La expresión práctica del desarrollo de una cultura histórica a partir del estudio de La Edad de Oro, como premisa para el desarrollo de una filosofía de la educación que estimula el conocimiento y la formación integral del ser humano, se concreta en la educación para la vida a partir de consejos, reflexiones críticas, comparaciones de procesos y hechos históricos vinculados con la ciencia, la naturaleza y el arte.

A través del Cuento "Bebé y el señor Don Pomposo", José Martí pone a pensar a los niños sobre la cultura de la época, sus matices y problemas. En recorrido de Raúl y Bebé por las calles de París:

han ido con su mamá a ver a los ciegos, que leen con los dedos, en unos libros con las letras muy altas: han ido a la calle de los periódicos, a ver como los niños pobres que no tienen casa donde dormir, compran diarios para venderlos después, y pagar su casa: han ido a un hotel elegante, con criados de casaca azul y pantalón amarillo, a ver a un señor muy flaco y muy estirado, el tío de mamá, el señor Don Pomposo. 
Bebé está pensando en la visita del señor Don Pomposo. Bebé está pensando. (J.M.O.C., T-18, 1975, p.346)

Se trata de una pedagogía de la ternura intencionada al desarrollo del pensamiento lógico, a la estimulación del razonamiento para entender que existe lo correcto, lo incorrecto, de qué lado se debe estar, y cómo actuar para transformar la realidad.

\section{Conclusiones}

Este puede ser el camino para la formación de las presentes y futuras generaciones en el amor por la vida, la historia, las causas justas, el respeto a la condición humana y todo el caudal axiológico que ello implica. En consecuencia, los educadores deben estar preparados para desarrollar en los educandos una cultura histórica humanista y renovadora desde el estudio de la Edad de Oro de José Martí. Con este fin, el esbozo de una concepción teórica marca las pautas para el ejercicio de un acto educativo centrado en el ser humano desde la impronta de José Martí, su universalidad y los nuevos caminos que se pueden transitar en la formación general.

\section{Referencias bibliográficas}

Bolet, N. (1971). José Martí como literato [Prólogo y notas de Gonzalo de Quesada y Miranda]. En Así vieron a Martí. La Habana, Cuba: De Ciencias Sociales.

Chávez, J. (2002). Prólogo. Turner, L. y Pita, B. (ed.). Pedagogía de la ternura. La Habana, Cuba: Pueblo y Educación.

Díaz, H. (1999). Cinco pautas Martianas para el profesor de historia. Pedagogía 99' Resúmenes, pp.161-175. La Habana, Cuba: Palcograf.

Díaz, H. (2006). Sobre la historia y otros temas. La Habana, Cuba: Pueblo y Educación.

Escribano, E. (2006). La concepción de la educación en la obra de José Martí. La Habana, Cuba: Pueblo y Educación.

Martí, J. (1959). La edad de oro. La Habana, Cuba: Gente Nueva.

Martí, J. (1975). Obras completas, (Tomos 1, 5, 6, 7, 8, 10, 13, 14, 15, 18, 19, 21, 22, 23, 27). La Habana, Cuba: De Ciencias Sociales. 
Martínez, M. (2003). La filosofía de la educación en José Martí: principios, direcciones, vigencia. En Blanco, A. (ed.). Filosofía de la educación. Selección de lecturas. La Habana, Cuba: Pueblo y Educación.

Pestana, Y. (2012, mayo 7). José Martí y su época. Una propuesta de tabla sincrónica. Revista Atenas. ISBN: 1628-2749.

Pestana, Y., Fajardo, M., Menéndez, E. y Diago, M. (2015). Sistematización de los fundamentos teórico-metodológicos para el desarrollo de la cultura histórica en la formación inicial del profesional de la educación en las Universidades de Ciencias Pedagógicas. En Proyecto de investigación El estudio de la obra Martiana en la formación humana del profesional de las ciencias pedagógicas. Matanzas, Cuba: Universidad de Matanzas.

Sabariego, M. (2002). La Educación Intercultural ante los retos del siglo XXI. Bilbao: Descleé.

Turner, L. y Pita, B. (2002). Pedagogía de la ternura. La Habana, Cuba: Pueblo y Educación.

Vitier, c. (2000, ene-mar). Martí en la Educación Superior. Honda, 1(1). La Habana, Cuba. 
